\title{
Clinicopathological Profile and Outcome of Acute Pancreatitis
}

\author{
M ARAHMAN ${ }^{\mathrm{a}}$, MUH BEGUM $^{\mathrm{b}}$, PK SHARMA ${ }^{\mathrm{c}}$, ATUL JHA ${ }^{\mathrm{d}}$, RAHUL JAIN $^{\mathrm{e}}$, SK SINGH $^{\mathrm{f}}$
}

Summary:

Background: Acute pancreatitis is an important cause of morbidity and mortality among gastrointestinal disorders. But little is known about etiology and clinical profile in Indian population.

Objective: To know clinicopathological profile, etiology and outcome of acute pancreatitis in study patients.

Material and methods: This observational cross-sectional study was conducted in a tertiary care and research hospital in New Delhi India from May 2018 to November 2018. Total 30 established cases of acute pancreatitis were included in the study. Data were collected and processed by using SPSS version 20 and result was obtained in tables and diagrams.

Results: Among 30 patients, 21(70\%) were male and 9(30\%) female; 18 to 89 yrs of aged patients were included in the study with mean age 41.6 \pm 17.5 years, of 18-30 years of aged patients were more affected (10,33.3\%); patients of different occupation were studied;26(86.7\%)patients were non-smoker and 4(13.3\%) smoker; 16(53.3\%) were non-alcoholic and 14(46.7\%) patients had a habit of alcoholism of whom, all were male; patients who used to take $e^{\text {" } 5}$ units of alcohol per

a. Lt Col Md Anisur Rahman, Classified Specialist in Medicine and Gastroenterologist, Dept. of Gastroenterology, Combined Military Hospital (CMH) Dhaka Cantonment.

b. Dr Most. Umme Habiba Begum, Associate Professor, Dept. of Paediatrics, Rangpur Community Medical College, Rangpur

c. Brig Praveen Kumar Sharmam, Head and consultant, Dept. of Gastroenterology, Army Hospital (Research and Referral), New Delhi India.

d. Lt Col Atul Jha, Associate Professor, Dept. of Gastroenterology, Army Hospital (Research and Referral), New Delhi India.

e. Lt Col Rahul Jain, Gastroenterologist, Command Hospital (NC) C/O 56 APO, India.

f. Lt Col Sudhir Kumar Singh, Associate Professor, Dept. of Gastroenterology, Army Hospital (Research and Referral), New Delhi India.

Address of Correspondence: Lt Col Md Anisur Rahman, Classified Specialist in Medicine and Gastroenterologist, Dept. of Gastroenterology, Combined Military Hospital (CMH) Dhaka Cantonment. Mobile: 01712278509, 01521-248085, Email: arafanis@gmail.com

Received: 18 July 2019

Accepted: 24 December 2019 day were frequently affected (10, $71.4 \%)$ by acute pancreatitis, though it did not spared occasional drinkers (2, 14.3\%);22(73.3\%) patients had interstitial pancreatitis and 8(26.7\%) had acute necrotizing pancreatitis;14(46.7\%) patients had acute pancreatitis due to alcohol, 10 (33.3\%) patients had gall stone, 2(6.7\%) patients developed pancreatitis after ERCP;29(96.7\%) patients presented with abdominal pain, 28 (93.3\%) had vomiting, 21 (70\%) patients had jaundice, 10 (33.3\%) had fever, 18 (60\%) patients had anemia, 17 (56.7\%) patients develop ascites, 19 (63.3\%) patients develop pleural effusion, 7(23.3\%) patients developed ileus, and 3(10.0\%) patients developed circulatory shock; 25(83.3\%) patients developed organ dysfunction during in hospital care, of whom 11(36.7\%) patients had transient and 14(46.7\%) had persistent organ dysfunction; 5(16.7\%) patients were complicated with pseudocyst, 6(20\%) had walled of necrosis (WON), 7(23.3\%) developed sepsis, 14(46.7\%) developed renal dysfunction, 23(76.7\%) developed hepatic dysfunction, 8(26.7\%) developed respiratory dysfunction, 6(20\%) developed pneumonia; 8(26.7\%) patients had been suffering from different comorbidity; ultrasound of abdomen were abnormal in all 30(100\%) patients; As per CTSI score, severe pancreatitis 14(46.7\%), moderate pancreatitis 14(46.7\%) and mild pancreatitis 2(6.7\%); 24(80\%) patients received only medical treatment and $6(20 \%)$ patients needed surgical or radiological intervention; 19(63.3\%) patients were improved symptomatically, $8(26.7 \%)$ patients were cured and 3(10\%) patients died during in hospital care.

Conclusion: Alcohol was the predominant etiology of acute pancreatitis, mostly affecting young and middle aged male, but mortality was more in gall stone related pancreatitis. Hepatic dysfunction was observed frequently that may attribute to effect of chronic alcohol abuse.

Key words: Acute pancreatitis, Clinicopathological profile, Alcohol pancreatitis, Gallstone pancreatitis, Outcome.

(J Bangladesh Coll Phys Surg 2020; 38: 86-92) DOI: https://doi.org/10.3329/jbcps.v38i2.45632

Introduction:

Acute pancreatitis is common and is a leading cause of hospitalization among gastrointestinal disorders. The diagnosis of acute pancreatitis (AP) requires at least two of the following features: characteristic abdominal pain; 
biochemical evidence of pancreatitis (i.e., serum amylase or lipase elevated $>3$ times the UNL); and/or radiographic evidence of pancreatitis on cross-sectional imaging ${ }^{1}$. Acute pancreatitis is divided into two pathological types: interstitial edematous pancreatitis and necrotizing pancreatitis. In industrialized countries, gallstones and alcohol abuse are the most frequent reasons for acute pancreatitis ${ }^{2}$.Both of these comprise about $80 \%$ of cases of acute pancreatitis. Gall stones cause approximately $40 \%$ of cases of acute pancreatitis ${ }^{3}$. However, only $3 \%$ to $7 \%$ of patients with gallstones develop pancreatitis. The remaining causes are less common, includes pancreatic malignancy, drug reactions, hypertriglyceridemia, post endoscopic retrograde cholangiopancreatography (ERCP), autoimmune, infections, trauma or surgery etc. The recent revised Atlanta classification has described acute pancreatitis into three distinct subtypes: mild (usually interstitial), moderately severe (local complications without persistent organ failure) and severe (persistent organ failure). Mild interstitial pancreatitis is the most commonly observed subtypes of acute pancreatitis and constitutes about $75-80 \%$ of all patients. Critical acute pancreatitis, a fourth class of severity, is described in the determinant-based classification ${ }^{4}$ when both infected necrosis and persistent organ failure are present together. Clinical presentation of acute pancreatitis varies with the severity of an attack. Common initial presentations are abdominal pain and tenderness with or without abdominal guarding, nausea, vomiting, tachycardia, tachypnea. Subsequently patients may be presented with fever, dyspnea, ileus, icterus, pleural effusions, ascites, hypotension or shock. There may be palpable epigastric mass due to pseudocyst or a large inflammatory mass. Uncommon presentations are ecchymosis in 1 or both flanks (Grey Turner's sign) or about the periumbilical area (Cullen's sign). Pain is absent in $5-10 \%$ of acute pancreatitis, and a painless presentation may be a feature of serious fatal disease ${ }^{5}$. There may be disorientation, hallucinations, agitation, or coma.

To establish a diagnosis of acute pancreatitis, revised Atlanta criteria ${ }^{1}$ is usually followed. After obtaining clinical information, common investigations required to establish diagnosis are serum amylase and or lipase and cross sectional imaging of abdomen such as computed tomography (CT) scan and or magnetic resonance imaging (MRI). The diagnosis of acute pancreatitis relies on at least a 3 -fold elevation of serum amylase or lipase in the blood. Conventional abdominal ultrasound scan should be performed in every patient with a first attack of acute pancreatitis to search for gallstones in the gallbladder, common duct stones, or signs of extra-hepatic biliary tract obstruction. Plain radiograph finding may range from no abnormalities in mild disease to localized ileus of a segment of small intestine ("sentinel loop") or the colon cut-off sign in more severe disease. In addition, an abdominal plain film helps to exclude other causes of abdominal pain, such as bowel obstruction or perforation ${ }^{6}$.Predicting severity of pancreatitis early in the course of disease is critical to maximize therapy and to prevent and minimize organ dysfunction and complications. There are different scoring systems used in different parts of world to predict severity of acute pancreatitis, such as Ranson's scoring, acute physiology and chronic health evaluation 11 (APACHE 11), bedside index of severity of acute pancreatitis (BISAP) scoring, Glasgow scoring etc.Imaging is used to assess severity of acute pancreatitis by using CT grading system of Balthazar and the CT Severity Index (CTSI) ${ }^{6}$.Modified CTSI (2004) includes sum of two scores: Balthazar scores- grading of pancreatitis (A: normal pancrease-0, B: enlargement of pancrease-1, $\mathrm{C}$ : inflammatory changes in pancrease and peripancreatic fats-2, D: ill-defined single peripancreatic fluid collection-3, E: two or more poorly defined peripancreatic fluid collections-4) and grading the extend of pancreatic necrosis (None: $0, \mathrm{~d}$ " $30 \%$ : 2 , $>30-50 \%: 4,>50 \%: 6$ ) with maximum score that can be obtained is 10. Balthazar score and those obtained with the evaluation of pancreatic necrosis are expressed as 0-2: mild acute pancreatitis, 4-6: moderate acute pancreatitis, 8-10: severe acute pancreatitis.

Patients with acute pancreatitis require early aggressive intravenous hydration to maintain hemodynamic stability and adequate perfuse of the kidneys and pancreas, adequate analgesia to eliminate or to reduce pain. Abdominal pain can be treated with opiate analgesics. Patients are usually kept nothing per oral (NPO) until any nausea and vomiting have subsided. Subsequent management of acute pancreatitis varies patient to patient depending on clinical scenario, underlying etiology and complications. Acute pancreatitis may be complicated with local effects like pseudocyst, pancreatic necrosis, duodenal perforation or bleeding and systemically, such as organ failure. Prognosis of acute pancreatitis depends on types of pancreatitis, severity, underlying etiology, co-morbidity, prediction of complications and appropriate intervention to combat those events. Overall mortality in acute pancreatitis is $10-15 \%$, but varies with different factors. Type 2 Diabetes mellitus has been associated with higher severity and mortality in the setting of acute pancreatitis? ${ }^{7}$. 


\section{Material and Methods:}

This study was conducted in Gastroenterology department of a tertiary care and research hospital in New Delhi India. Data were collected during the period of May 2018 to November 2018. Verbal consents from the patients or relatives were taken. It was a cross sectional observational study. The study was aimed to include acute pancreatitis cases of any age, sex, etiology or any physical condition like pregnancy. Total 30 established cases of acute pancreatitis, as per revised Atlanta criteria were included in the study. Exclusion criteria were chronic or recurrent acute pancreatitis cases or suspected/doubtful cases of acute pancreatitis. Transfer out case was also excluded from the study. Verbal consent was taken from patient or relative and physical examination of each patient was done. Data was collected and analysis was done by using SPSS version 20.Results were obtained in tables and diagrams. As there was no involvement of invasive procedure or intervention ethical clearance was not taken.

\section{Result:}

\section{Table-I}

\begin{tabular}{lcc}
\multicolumn{2}{c}{ Demographic profile of study patients $(n=30)$} \\
Traits & Frequency & Percentage \\
\hline Age & & \\
Mean \pm SD & $41.6 \pm 17.5$ & \\
Minimum & 18 & \\
Maximum & 89 & \\
18-30yrs & 07 & 23.3 \\
31-45yrs & 10 & 33.3 \\
46-60 yrs & 08 & 26.7 \\
Above 60 yrs & 05 & 16.7 \\
Gender & & \\
Male & 21 & 70 \\
Female & 09 & 30 \\
Occupation & & \\
Service & 11 & 36.7 \\
Housewife & 09 & 30.0 \\
Retired from service & 06 & 20.0 \\
Student & 04 & 13.3 \\
Smoking habit & & \\
Yes & 4 & 13.3 \\
No & 26 & 86.7 \\
Alcohol intake & & \\
Yes & 14 & 46.7 \\
Male & 14 & 46.7 \\
Female & - & - \\
No & 16 & 53.3 \\
\hline
\end{tabular}

Out of 30 patients, 21 were male $(70 \%)$ and 9 were female $(30 \%)$; 11 patients were service $\operatorname{man}(36.7 \%), 9(30 \%)$ were house wife, $6(20 \%)$ were retired from service and remaining 4(13.3\%)were students; among all patients 26(86.7\%)were non-smoker and $4(13.3 \%)$ were smoker; $14(46.7 \%)$ patients had a habit of alcoholism, all were male and $16(53.3 \%)$ were non-alcoholic.

Table-II

Etiology and types of acute pancreatitis $(n=30)$

\begin{tabular}{ccc} 
Characters & $\begin{array}{c}\text { Number } \\
(\mathrm{n}=30)\end{array}$ & Percentage \\
\hline
\end{tabular}

Types of pancreatitis

\begin{tabular}{lcc} 
Acute interstitial & 22 & 73.3 \\
$\begin{array}{l}\text { Acute necrotizing } \\
\text { iology of Pancreatitis }\end{array}$ & 08 & 26.7 \\
Alcohol & 14 & 46.7 \\
Gall stone & 10 & 33.3 \\
Post ERCP & 2 & 6.7 \\
Autoimmune & 1 & 3.3 \\
Others (Hypercalcaemia) & 1 & 3.3 \\
Idiopathic & 2 & 6.7 \\
\hline
\end{tabular}

This table shows, among 30 patients of acute pancreatitis $22(73.3 \%)$ had interstitial pancreatitis and $8(26.7 \%)$ had acute necrotizing pancreatitis; fourteen patients (46.7\%) were alcoholic, $10(33.3 \%)$ patients had gall stone,2(6.7\%)patients developed pancreatitis after ERCP, 1(3.3\%) of each had autoimmune pancreatitis and hypercalcaemia (primary hyperparathyroidism) and $2(6.7 \%)$ were of unknown etiology.

\section{Table-III}

\section{Clinical presentation of study patients of acute pancreatitis $(n=30)$}

\begin{tabular}{lcc} 
Characters & Frequency & Percentage \\
\hline Abdominal pain & 29 & 96.7 \\
Vomiting & 28 & 93.3 \\
Jaundice & 21 & 70.0 \\
Pleural effusion & 19 & 63.3 \\
Anemia & 18 & 60.0 \\
Ascites & 17 & 56.7 \\
Fever & 10 & 33.3 \\
Ileus & 07 & 23.3 \\
Circulatory shock & 03 & 10.0 \\
\hline
\end{tabular}


Twenty nine of study patients $(96.7 \%)$ of acute pancreatitis presented with abdominal pain, $28(93.3 \%)$ had vomiting, 21 (70\%) patients had jaundice, 19(63.3\%) patients develop pleural effusion, 18(60\%) patients had anemia, $17(56.7 \%)$ patients develop ascites, $10(33.3 \%)$ had fever, 7(23.3\%) patients developed ileus, and 3(10\%) patients developed circulatory shock.

\section{Table-IV}

\begin{tabular}{|c|c|c|}
\hline \multicolumn{3}{|c|}{$\begin{array}{l}\text { Profile of organ dysfunction of } \\
\quad \text { study patients }(n=30)\end{array}$} \\
\hline Characters & Frequency & Percentage \\
\hline \multicolumn{3}{|l|}{ Organ dysfunction } \\
\hline Yes & 25 & 83.3 \\
\hline No & 05 & 16.7 \\
\hline Transient organ dysfunction & 11 & 36.7 \\
\hline Persistent organ dysfunction & 14 & 46.7 \\
\hline Total & 30 & 100 \\
\hline
\end{tabular}

Out of 30 patients, $25(83.3 \%)$ developed organ dysfunction and 5(16.7\%) had none during in hospital care; $11(36.7 \%)$ patients had transient organ dysfunction and $14(46.7 \%)$ had persistent organ dysfunction.

\section{Table-V}

\begin{tabular}{lcc}
\multicolumn{3}{c}{$\begin{array}{c}\text { Co-morbidities in acute pancreatitis } \\
\text { patients }(n=30)\end{array}$} \\
Co-morbidity & Number $(\mathrm{n}=30)$ & Percentage \\
\hline Hypertension & 2 & 6.7 \\
Autoimmune disorder & 2 & 6.7 \\
Diabetes mellitus & 1 & 3.3 \\
Chronic kidney disease & 1 & 3.3 \\
Coronary arterial disease & 1 & 3.3 \\
Multiple co-morbidity & 1 & 3.3 \\
\hline Total & 8 & 26.7 \\
\hline
\end{tabular}

Out of 30 study patients $8(26.7 \%)$ had been suffering from different co-morbidities like hypertension, Diabetes mellitus, chronic kidney disease, autoimmune disorders, Coronary artery disease etc; one $(3.3 \%)$ patient had multiple co-morbidities.
Table-Vl

Complications of study patients $(n=30)$

\begin{tabular}{lcc} 
Characters & Number $(\mathrm{n}=30)$ & Percentage \\
\hline Liver dysfunction & 23 & 76.7 \\
Renal dysfunction & 14 & 46.7 \\
Respiratory dysfunction & 08 & 26.7 \\
Sepsis & 07 & 23.3 \\
Pneumonia & 06 & 20.0 \\
Walled of necrosis (WON) & 06 & 20.0 \\
Pseudocyst & 05 & 16.7 \\
Circulatory dysfunction & 03 & 10.0 \\
Gastrointestinal bleeding & 03 & 10.0 \\
DIC & 02 & 6.7 \\
\hline
\end{tabular}

\# Liver dysfunction- raised ALT $\geq 2$ times of normal upper limit/ previous result; Renal dysfunction- raised serum creatinin $0.3 \mathrm{mg}$ from base line/ normal upper limit.

Among 30 study patients, 23(76.7\%)developed hepatic dysfunction, 14(46.7\%) developed renal dysfunction, $6(20 \%)$ had walled of necrosis (WON), $8(26.7 \%)$ developed respiratory dysfunction, $7(23.3 \%)$ were complicated by sepsis, 6(20\%) developed pneumonia,5(16.7\%) were complicated with pseudocyst, $3(10 \%)$ developed circulatory dysfunction, $3(10 \%)$ developed gastrointestinal bleeding and $2(6.7 \%)$ developed DIC.

\section{Table-VII}

Imaging profile of study patients $(n=30)$

Modalities Number $(\mathrm{n}=30) \quad$ Percentage

Ultrasound

$\begin{array}{lll}\text { Abnormal } & 30 & 100.0\end{array}$

CT severity index (CTSI)

$0-2$ (mild)

4-6 (moderate)

46.7

$8-10$ (severe)

\begin{tabular}{lll}
\hline Total & 30 & 100 \\
\hline
\end{tabular}

Table shows,imaging profile of study patients, ultrasound of abdomen was abnormal in all 30(100\%) patients; computed tomography severity index (CTSI) score were $0-2$ (mild) in 2(6.7\%) patients, 4-6 (moderate) in $14(46.7 \%)$ patients and $8-10$ (severe) in $14(46.7 \%)$ patients. 
Medical

combine, medical and

$\square$ surgical/Radiologica intervention

Treatment modality received

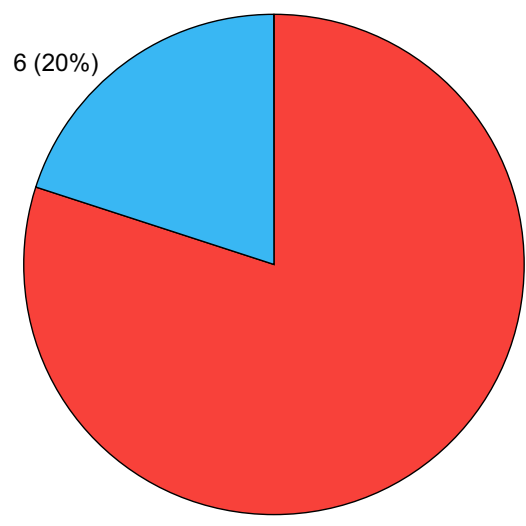

$24(80 \%)$

Fig.-1: Modalities of treatment received by study patients $(n=30)$

Diagram shows, out of 30 patients $24(80 \%)$ received only medical treatment and remaining $6(20 \%)$ patients needed surgical and or radiological intervention to treat complications like pseudocyst or walled of necrosis.

\section{Table-VIII}

Outcome of acute pancreatitis patients $(n=30)$

Traits Number $(n=30) \quad$ Percentage

Outcome of inhospital care

$\begin{array}{lll}\text { Cured } & 08 & 26.7\end{array}$

Improved $\quad 19 \quad 63.7$

$\begin{array}{lll}\text { Expired } & 03 & 10.0\end{array}$

$\begin{array}{lll}\text { Gall stone related } & 2 & 6.7\end{array}$

\begin{tabular}{lcc} 
Alcohol related & 1 & 3.3 \\
\hline Total & 30 & 100
\end{tabular}

\# Cured: symptom free/noreporting to hospitalwithin 3 months of discharge; Improved: had a few symptom/needed readmission within 3 months of discharge.

Among 30 acute pancreatitis patients, 08(26.7\%) were cured, $19(63.7 \%)$ were improved during inhospital care and $03(10.0 \%)$ patients expired of whom $2(6.7 \%)$ were gall stone pancreatitis and 01 (3.3\%) were alcohol related pancreatitis.

\section{Discussion:}

Acute pancreatitis defines an inflammatory process of the pancreas ${ }^{8}$ where inflammation can remain localized to the gland, or can involve other regional tissues or distant organ systems. It accounts for 3\% of all cases of abdominal pain ${ }^{9}$. The annual incidence of acute pancreatitis in Taiwan ranges from 13 to 45 per 100,000 persons ${ }^{10}$ and in United States ${ }^{11}$ it is approximately 40 cases per year per 100,000 adults. Nationwide incidence of acute pancreatitis in India is not known.

Our study observed interesting etiological profile pattern of acute pancreatitis. We found alcohol $(14,46.7 \%)$ was the predominant cause of acute pancreatitis, next common cause was gall stone (33.3\%) and idiopathic $6.7 \%$. In previous study 12 in Eastern India it was also observed that the commonest etiology of acute pancreatitis was attributed to alcohol (29.4\%), idiopathic $20.5 \%$ and obstructive cause (gall stone) $14.5 \%$.In other study at the All India Institute of Medical Sciences (AIIMS) ${ }^{13}$ observed gall stone and alcohol was the most common cause of acute pancreatitis in India, gall stone $(60 \%)$ being twice as common as alcohol $(30 \%)$.

In our study, another interesting observation was that acute pancreatitis were predominantly in male $(70 \%)$ whereas in female $30 \%$ and affected all age groups (1889 years) of people, though it was predominant in 31 to 45 years of age group $(10,33.3 \%)$. The risk of acute pancreatitis progressively increases with age and equal proportion of men and women are affected ${ }^{14}$. Alcohol related pancreatitis is more common in men, though sex differences disappear with similar level of alcohol consumption ${ }^{15}$. We found most of the alcohol related acute pancreatitis developed in heavy drinkers though it did not spared occasional drinkers and all the patients of acute pancreatitis of this etiology were male $(100 \%)$. The type of disease severity in acute pancreatitis has had a major influence on mortality. The overall mortality was relatively high $(21.1 \%)$ in a study ${ }^{16}$. Another study from Eastern European countries ${ }^{17}$ showed the complications of acute biliary pancreatitis was more serious and mortality rate was $15.1 \%$ in male and $17.8 \%$ in female. In our study we found overall mortality $10 \%$ and among severe acute pancreatitis it was $21.4 \%$ (3 of 14). All the patients who died had severe acute pancreatitis and were complicated with multi-organ failure of whom $2(6.7 \%)$ was gall stone pancreatitis, 1 (3.3\%) alcohol related and all three patients died during early phase of inhospital care.

Acute pancreatitis may be complicated and result in local or systemic problems. The systemic complications 
are usually seen in acute severe pancreatitis. Common complications are pulmonary oedema, sepsis, renal dysfunction, hepatic dysfunction, gastrointestinal bleeding, colitis and splenic vein thrombosis, pancreatic pseudocysts etc. We observed that hepatic dysfunction, transient or persistent were more common(23, 76.7\%) and next common was renal(14, $46.7 \%$ ) dysfunction, including one patient who needed haemodialysis for prolong period ( $>40$ sessions). The causes for renal dysfunction or acute kidney injury (AKI) in severe acute pancreatitis are multi-factorial, such as systemic inflammation, cytokine production, free radicals and other factors influencing the renal microcirculations. In a previous study ${ }^{18}$ in India AKI was found $19.4 \%$ in acute pancreatitis and incidence rate may ranges from $14 \%$ to $42 \%$. Pancreatic pseudocysts are also common in acute pancreatitis. It arises from pancreatic ductal disruption. It occurs in $10 \%$ to $26 \%$ of acute pancreatitis cases ${ }^{19}$. We also found pancreatic pseudocysts in 5(16.7\%) cases in our study. Secondary pancreatic infection and Sepsis is common in acute pancreatitis. It is caused by bacterial contamination of pancreatic necrosis. The incidence of secondary infection and sepsis correlates with the extent of pancreatic necrosis. It may develop in $40-70 \%$ of patients with $80 \%$ mortality $^{20}$. In our case, sepsis was found in $7(23.3 \%)$ patients. The prevention of infection and sepsis by systemic administration of antibiotics is considered a principal step in the therapy of acute pancreatitis along with other symptomatic and supportive treatments.

\section{Limitation of the study:}

This study was done in a research and referral hospital in Delhi city of India in small number of patients and it may not reflect the total scenario of acute pancreatitis cases admitted in hospitals all over the country.

\section{Conclusion:}

Alcohol was the predominant etiology of acute pancreatitis, mostly affecting young and middle aged male, but mortality was more in gall stone related pancreatitis. Hepatic dysfunction was observed frequently that may attribute to chronic alcohol abuse in alcohol related pancreatitis cases. However, further large scale study for extended period and national data base are required to formulate complete picture of acute pancreatitis in Indian nation.

\section{Conflict of Interest: None declared.}

\section{Reference:}

1. BanksP A, BollenTL, Dervenis C, Gooszen HG, Johnson CD, Sarr MG. Classification of acute pancreatitis -2012: revision of Atlanta classification and definition by international concensus. GUT.2013;62: 102-11.

2. Frossard JL, Steer ML, Pastor CM, Acute pancreatitis. Lancet 2008;371: 143.

3. Rosendahl J, Bernadova J, Olfert L, Kovacs P, Teich N, Bodeker H. CFTR, SPINK1, CTRC and PRSS1 variants in chronic pancreatitis: is the role of mutated CFTR overestimated? GUT 2013;62:582.

4. Dellinger EP, Forsmark CE, Layer P, et al. Determinant based classification of acute pancreatitis severity: an international multidisciplinary consultation. Ann Surg 2012;256:875-80.

5. Lankisch PG, Schirren CA, Kunze E. Undetected fatal acute pancreatitis: Why is the disease so frequently overlooked? Am J Gastroenterol 1991; 86:322-6.

6. Scott Tenner and William M. Steinberg. Acute Pancreatitis. Sleisenger and Fordtran's Gastrointestinal and liver disease. $10^{\text {th }}$ edn. $58 ; 969-93$.

7. Huh JH, Jeon H, Park SM, Choi E, Lee GS, Kim JW, Lee $\mathrm{KJ}$; Diabetes Mellitus is associated with mortality in acute pancreatitus. J Clin Gastroenterol. 2018; 52(2):178-83.)

8. Kloppel G, Dreyer T, Willemer S, Horst F, Guido A;Human acute pancreatitis: its pathogenrsis in the light of immunocytochemical and ultrastructural finding in acinar cell. Virchows Arch A Pathol Anat Histopathol. 1986; 409: 791.

9. I.D Penman, C.W. Lees; Alimentary tract and pancreatic disease; Davidson's Principle and practice of medicine; $22^{\text {nd }}$ edn; 22;837-920.

10. Shen HN, Lu CL, Li CY. Epidemiology of first attack acute pancreatitis in Taiwan from 2000 through 2009: a nationwide population based study. Pancrease. 2012;41: 696-702.

11. Granger J, Remick D. Acute pancreatitis: models, markers, and mediators. Shock.2005; 24(1)45-51.

12. Dibyndu M, Shantnu B, Sandeep L, Rajasree S; Demographic profile of acute pancreatitis in eastern aiandia: a single center experience. Asian J of Med. Sciences. 2017;8(6): 24-8.

12. Tandon RK. Management of acute pancreatitis: Indian guidelines and protocol. API Medicine Update2013;23:267270 .

14. Dhiraj Yadav, Albrt B, Lowenfels.The Epidemiology of Pancreatitis and Pancreatic Cancer. Gastroenterol.ogy. 2013;144(6)1252-61.

15. Lankisch PG, Lowenfels AB, Maisonneuve P. What is the risk of alcoholic pancreatitis in heavy drinkers? Pancrease. 2002; 25: 411-2. 
16. Popa CC, Badiu DC, Rusu OC, Gregorian VT, Neagu SI, Strugaru CR. Mortality

prognostic factors in acute pancreatits. J Med Life.2016;9(4):413-8.

17. Katan R, Posan J, Sapy P, Damjanovich L, Szenthereszty Z. Analysis of clinical course of severe acute biliary and non-biliary pancreatitis : a comparative study. OrvHtil. 2010;151(7): 265-8.
18. Ravindra K, Naresh P, Neeraj J. Acute kidney injury in severe acute pancreatitis: an experience from a tertiary care centre. Saudi J Kidney Dis Transpl 2015;26:56-60.

19. Andrew L, Samuelson MD, Raj J, Shah MD. Endoscopic management of pancreatic pseudocysts. Gastroenterology clinics of North America. 2012; 41(1): 47-62.

20. Mifkovic A, Pindak D, Daniel I, Pechan J. Septic complications of acute pancreatitis. Bratisl Lek Listy.2006;107(8):296-313. 